Mots. Les langages du politique

\title{
Ennemis, hôtes et étrangers. Enquête sur les identités politiques grecque et romaine
}

Inimigos, hóspedes e estrangeiros. Estudo sobre as identidades políticas gregas e romanas

Enemies, hosts and foreigners. Survey of Greek and Roman political identities Enemigos, huéspedes y extranjeros. Encuesta sobre las identidades griegas y romanas

\section{Benjamin Boudou}

\section{OpenEdition}

Journals

Édition électronique

URL : https://journals.openedition.org/mots/21218

DOI : $10.4000 /$ mots. 21218

ISSN : 1960-6001

\section{Éditeur}

ENS Éditions

\section{Édition imprimée}

Date de publication : 22 avril 2013

Pagination : 127-140

ISBN : 978-2-84788-393-0

ISSN : 0243-6450

\section{Référence électronique}

Benjamin Boudou, «Ennemis, hôtes et étrangers. Enquête sur les identités politiques grecque et romaine ». Mots. Les langages du politique [En ligne], 101 | 2013, mis en ligne le 22 avril 2015, consulté le 22 avril 2022. URL : http://journals.openedition.org/mots/21218; DOI : https://doi.org/10.4000/ mots. 21218 


\section{Ennemis, hôtes et étrangers. Enquête sur les identités politiques grecque et romaine}

Il existe un ancien problème en apparence banal, une argutie de linguistes et d'historiens qui n'intéresse que les antiquisants, ayant au mieux nourri une prose philosophique bavarde : le mot «étranger» a fini, en latin, par désigner l'« ennemi ». Sa formulation la plus simple apparaît chez Cicéron (1974, l-12) :

La tristesse de la réalité a été atténuée par la douceur du terme, en ceci que fut appelé hostis, celui qui à proprement parler était perduellis [ennemi]. On nommait hostis en effet chez nos aïeux, celui que maintenant nous nommons peregrinus, étranger.

Cicéron semble ne faire qu'enregistrer une évolution linguistique, un changement de sens certes étonnant mais pas anormal. Il avance l'hypothèse d'une sorte de politesse ou de mansuétude, à l'égard de l'ennemi, qui aurait atténué la violence de perduellis (dont le préfixe per-est à la fois intensif et péjoratif). L'usage aurait par la suite rechargé ce «doux terme» de la valeur négative de perduellis tombé en désuétude (Gallego, 2010). L'affaire a pris un tour plus complexe, quand Émile Benveniste a souligné l'intrication étymologique de l'hostis avec l'hospes (l'hôte), puis comparé le tout au xénos grec, lui aussi oscillant entre l'hôte et l'étranger, mais sans lien avec l'ennemi (Benveniste, 1969). Si nous ne prétendons pas résoudre cette énigme linguistique, déjà floue et controversée du temps de ses contemporains, il est néanmoins possible d'en proposer une interprétation politique. Cette contamination de l'étranger et de l'hôte par l'ennemi semble en effet au cœur d'un enjeu plus large : hostilité et hospitalité se trouvent rassemblées autour du problème politique de la définition de «l'autre», avec qui on engage ou non la réciprocité, déterminant ainsi la notion de citoyenneté ${ }^{1}$. Nous allons comprendre pourquoi l'ennemi doit d'abord être fait étranger, et comment l'hôte permet de

1. Cet usage «contaminé » de l'étranger et de l'ennemi est caractéristique chez Carl Schmitt par exemple (1992, p. 64) : «L'ennemi politique ne sera pas nécessairement mauvais dans l'ordre de la moralité ou laid dans l'ordre esthétique [...]. Il se trouve simplement qu'il est l'autre, l'étranger. »

Institut d'études politiques de Paris, CEVIPOF

benjamin.boudou@sciences-po.org 
qualifier un étranger intermédiaire, en relation à la cité sans en être membre. Nous en tirerons des définitions et critères pour l'étude des modes d'appartenance politique.

\section{Problèmes et hypothèses}

Plusieurs auteurs ont avancé des hypothèses sur les évolutions institutionnelles des cités grecques ou romaines pour expliquer ces problèmes de vocabulaire. Mais à vouloir travailler par exemple à partir d'une histoire des paires conceptuelles antonymes - entre Grecs et Barbares, ou entre citoyens et ennemis, et citoyens et étrangers - à la manière de Reinhart Koselleck (1990), on ne peut que buter sur le statut conceptuel et historique de l'hôte (l'invité, celui qui est en relation d'hospitalité). Mieux vaut alors suivre plus doucement les relations tissées entre une cité et ses multiples autres, comme le fait de Georges Dumézil (1977) à propos de l'arì védique, lui aussi oscillant entre ennemi et étranger ${ }^{2}$. Ces relations (incluant l'hostilité) aux non-citoyens dessinent en creux la conception du collectif, c'est-à-dire le sens de la communauté politique. Elles permettent également de dépasser la double entrée usuelle intégration-exclusion qui demeure insuffisante pour comprendre le sens politique de l'hospitalité à Athènes et Rome. Par cette approche «relationniste», on se donne les moyens d'analyser les «identités politiques» de ces communautés, et de les rendre heuristiques par-delà le contexte ancien. Par identité politique, il faut entendre les modalités d'appartenance d'une communauté, «la manière dont ses membres lui appartiennent (et dont ils appartiennent à d'autres groupes) » (Maier, 1994, p. 19-20). En choisissant comme critère d'analyse la relation aux étrangers, nous cherchons donc à déterminer leur place dans la construction d'une communauté politique, plus particulièrement à partir des institutions grecques et latines de l'hospitalité. L'hypothèse principale est qu'il n'est pas anodin d'associer «ennemi » et «étranger», non seulement dans la rhétorique politique, mais surtout dans la compréhension même qu'on a de la cité, de ses frontières et, bien sûr, de la citoyenneté.

Cette indistinction entre ennemi, hôte et étranger a certainement un fondement anthropologique largement partagé, l'altérité de l'étranger étant une source d’inquiétude qui met en jeu l'identité de la communauté (Boudou, 2012). Or il s'agit déjà d’un enjeu politique, caril devient un enjeu relationnel

2. Son interprétation est d'ailleurs proche de la nôtre, puisqu'il démontre que cette ambiguïté témoigne en fait d'une double classe d'étrangers : ceux avec qui il peut y avoir relations (de l'hospitalité à l'hostilité) mais qui ne sont pas membres du groupe social restreint, d'une part, et les autres, radicalement hostiles, d'autre part. Sa découverte la plus féconde est sans doute que l' "étranger», au sens générique, est une «notion moderne », un « fantôme tardif de l'esprit humain », qui était en fait d'abord qualifié. 
quand il fait institution, c'est-à-dire quand il produit des distinctions en droit ou des relations de pouvoir réciproque. Pour illustrer cela simplement, l'étranger grec (xénos) va d'abord s'opposer à l'endemos («l'homme du dedans, celui qui est ici»), puis au polites (le «citoyen») (Gauthier, 1973, p.4) : autrement dit, d'une relation de fait qui distingue l'intérieur de l'extérieur à une relation politique qui sépare le citoyen de l'étranger, se dessine la mise en forme politique d'un problème anthropologique.

Benveniste a donc bien posé le problème 3 : sa thèse principale est de souligner le sens premier d'hostire, signifiant égaliser, compenser, l'hostis étant d'abord quelqu'un avec qui l'on entretient ou institue une relation de réciprocité, voire d'égalité - deux choses différentes que Benveniste semble parfois confondre. Si hostis compose le mot "hôte» (hospes), c'est que l'étranger n'est pas le simple non-citoyen, il est «l'étranger, en tant qu'on lui reconnaît des droits égaux à ceux des citoyens romains», "celui qui est en relation de compensation» (Benveniste, 1969, p. 93-94). Plus précisément, l'hospes est un étranger à Rome, «placé sous la protection d'un citoyen romain à qui il rendra le même service quand ce dernier se rendra dans sa propre cité» (Gallego, 2010, p. 333). L'hospitalité correspond donc au mode positif de la réciprocité, l'hostilité à son mode négatif. Les sens d'hôte et d'ennemi se distinguent ainsi, comme les deux faces de la même réciprocité4.

Mais l'énigme demeure, car hostis a fini par prendre définitivement le sens d'ennemi, se détachant radicalement de l'hospes. L'hypothèse principale d'Émile Benveniste - qui reste à l'état d'hypothèse - est que l'institution de l'hospitalité évolue des relations privées aux relations publiques, en accompagnant la construction de Rome comme cité politique :

À date historique, l'institution [de l'hospitalité] avait perdu de sa force dans le monde romain : elle suppose un type de relations qui n'était plus compatible avec le régime établi. Quand l'ancienne société devient nation, les relations d'homme à homme, de clan à clan, s'abolissent; seule subsiste la distinction de ce qui est intérieur ou extérieur à la civitas. (Benveniste, 1969, p. 95)

3. Si bien d'ailleurs qu'il est repris tel quel dans la plupart les travaux sur l'hospitalité. Jacques Derrida a par exemple témoigné de sa dette envers le linguiste en forgeant le mot-valise « hostipitalité » pour souligner la composante hostile de l'hospitalité. Il rappelle également à plusieurs reprises (Derrida, 1996, 1997; Derrida, Dufourmontelle, 1997) la composition étymologique de l'hospitalité, hosti-pet, non pas «maître de l'hôte», comme il l'écrit en lisant trop vite Benveniste, mais l'hôte «lui-même », celui qui reçoit, c'est-à-dire qui incarne ou représente l'hospitalité (Benveniste, 1969, p. 95).

4. L'hypothèse peut d'ailleurs se démontrer avec une prémisse analytique différente : Julie Gallego (2010, p. 334-336) reprend avec clarté l'analyse des catégories pronominales de Françoise Bader, selon laquelle hospes et hostis sont tous les deux des dérivés d'une même racine indo-européenne qui donna également le xénos grec $\left(g^{\mathrm{h}} \mathrm{O}\right.$-s-ti donnant hostis, $g^{\mathrm{h}} \mathrm{O}$-s-pet donne

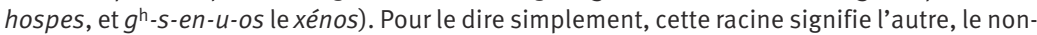
soi ; les deux formes désignent ainsi deux types de relation, ou bien l'autre qu'on n'accepte pas (hostis), ou bien l'autre qu'on accueille (hospes). 
D’un point de vue plus anthropologique, Marcel Hénaff reprend ce raisonnement. L'étranger devient celui avec qui les rapports de réciprocité (notamment de parenté) deviennent impossibles : «[l'hostis] devint donc un étranger, ce qui signifie en effet : un ennemi en puissance» (Hénaff, 2002, p. 196). Mais cette idée rencontre deux écueils majeurs : d'abord, l'hostilité n'empêche pas nécessairement la réciprocité, ce serait là une réduction de sens. La reconnaissance de l'autre comme ennemi est aussi une reconnaissance, précisément une relation juridique ouvrant la possibilité d'une guerre juste (Grangé, 2003). Ensuite, la naissance de la civitas n'a pas mis fin aux relations privées, l'hospitalité demeure; il n'y a donc pas de raison évidente pour que hostis se radicalise.

\section{Les mots en guerre contre la guerre}

Pour résoudre cette énigme et proposer notre hypothèse d'une conjuration de la guerre entre citoyens dans les mots mêmes, deux éléments de comparaison entre le xénos grec et l'hostis romain doivent être posés au préalable. D'abord, il faut se demander pourquoi xénos ne se radicalise pas en ennemi, comme c'est le cas de hostis. En effet, les transformations de l'hostis apparaissent d'autant plus étonnantes que le xénos - signifiant à la fois « hôte» et «étranger», déjà du temps d'Homère - ne prend jamais une tournure hostile (Gauthier, 1973). Cette double signification correspond à un fait grec essentiel : l'étranger xénos ne peut être que l'étranger avec qui la xenia (l'alliance, l'hospitalité) est possible. En somme l'étranger grec, hôte potentiel, ne peut être qu'un autre Grec, reconnu par le partage de la langue et des dieux. Autrement dit, parce que les conditions de possibilité d'une alliance sont la réciprocité et la permutabilité des rôles, seul un étranger largement familier peut la contracter5. Le fossé radical entre Grecs et Barbares, ou dans la période archaïque entre xénos et allotrioi (l'étranger sans réciprocité, commerçant ou pirate), permet la qualification plus fine d'étrangers grecs permettant de découpler l'étranger de l'ennemi. Cet étranger intermédiaire, ni totalement étranger ni totalement familier, est très précisément celui avec qui l'hospitalité est possible; le xénos sera donc ou étranger ou hôte, mais pas ennemi.

Deuxième élément de comparaison : c'est la distinction entre privé et public, plus exactement entre relations individuelles et relations communautaires, qui permet de mieux comprendre comment hostis et xénos ont pu avoir

5. Cette idée a été contredite par Gabriel Herman (1987, p. 12), qui ne constate pas de limite particulière à la xenia. On peut objecter que cette "amitié-hospitalité » ayant comme caractéristique d'être « ritualisée » (l'auteur parle de « ritualised friendship »), cela implique une référence transcendante qui doit l'être pour les deux parties afin d'être effective (c'est-à-dire afin de faire respecter les termes d'un accord non juridique). 
un destin politique différent ${ }^{6}$. En effet, l'hostis désigne d'abord l'étranger en relation de réciprocité, un étranger résident, sujet de droit; c'est un individu en rapport avec une communauté, la relation est publique. L'hospes constitue l'équivalent pour des relations entre individus (ou familles), bref pour désigner une relation privée. Ces deux types de relation ont pu être confondues à l'époque archaïque, ce qui expliquerait la contamination étranger-hôte (hostis-hospes), mais ils ont fini par se séparer. Même contamination pour le xénos, puis même évolution de la xenia privée ou familiale vers la proxenia communautaire ou politique, témoignant d'une séparation entre des relations privées individuelles et des conventions juridiques. Mais la différence essentielle, c'est la hiérarchie entre privé et public : tandis que chez les Grecs, la relation personnelle est la condition de possibilité de la relation politique, chez les Romains, l'hospitalité publique est la condition d'une reconnaissance privée. Les relations inter-cités ayant été plus courantes, la condition pour être hospes est d'être d'abord hostis (Gauthier, 1973, p. 19), la relation publique prévaut.

Le xénos conserve une ambiguïté entre privé et public, tandis que l'hostis se sépare plus clairement de l'hospes, la relation publique devenant plus déterminante que la relation privée. La question est donc désormais : en quoi cela a-t-il permis à l'hostis de dériver vers l'ennemi ? Comment l'étranger à la communauté devient-il a priori un ennemi? Nous pensons que la pensée politique nous permet de formuler l'hypothèse suivante : parce qu'il était impossible qu'un ennemi fût un membre. L'hostis prend le sens d'ennemi parce que l'ennemi ne peut être qu'un étranger. Et si le xénos ne change pas, c'est parce que, comme nous l'avons évoqué, le xénos n'est jamais qu'un autre Grec, un étranger qui n'en est pas vraiment un, et que la xenia est trop enracinée dans la sphère privée. La transformation de l'étranger en ennemi correspond à la définition même de l'identité politique que les Anciens se donnent : il ne peut y avoir guerre qu'avec des étrangers, car une communauté n'aurait pas de sens si elle était divisée. Et si l'ennemi est intérieur, c'est qu'il est en fait étranger.

De nombreux travaux ont démontré ce refoulement de la guerre civile, dessinant en creux le sens même de la politique comme fabrique constante, si ce n'est d'unité, au moins de cohésion :

La cité en guerre est ce contre quoi toute la philosophie politique s'est construite. [...] Le politique en général est ordonné autour de la conjuration de la guerre civile [...]. Il y a un refus de la nommer [...]. Conjurer revient à vouloir extérioriser ce qui est intérieur, dramatiser la violence du conflit civil pour le rendre tabou. (Grangé, 2009, p. 42-46)

C'est précisément pour cette raison que les mots mêmes en sont venus à participer à cette guerre contre la guerre. C'est ainsi par exemple que Platon

6. Encore une fois, l'ennemi (hostis) se séparant de l'hôte (hospes) et de l'étranger (peregrinus), le xénos gardant l'ambiguïté hôte-étranger sans signifier l'ennemi (echtros). 
justifie la fameuse distinction entre polemos et stasis, dessinant «la figure idéale de la polis, guerrière au-delà de ses portes, caractérisée en son sein par la paix civile» (Loraux, 1997, p. 27) :

De la même façon que nous disposons de deux noms pour nommer cela - guerre [polemos] et dissension [stasis] -, il existe également deux réalités [...]. Quand je parle de ces deux réalités, je veux dire d'une part ce qui est apparenté au même peuple et lui appartient, et de l'autre le différent et l'étranger. Or, à l'hostilité à l'endroit du proche [oikeoi] on donne le nom de dissension [stasis], à celle qui se porte contre ce qui est différent [allotrioi - étranger] on donne le nom de guerre [polemos]. (Platon, 2008, V-470b)

Notons que l'étranger contre qui on fait la guerre est allotrioi, c'est-à-dire l'étranger-ennemi, «qui parle[nt] d'autres langues», celui avec qui « les seules relations possibles sont de commerce (ou de piraterie)» (Gauthier, 1973, p. 6). Il ne s'agit pas du xénos qui, lui, est un autre Grec, partageant le sentiment « de n'être jamais totalement “extérieur” d'une cité à l'autre » (Vatin, 1984, p. 144). Le parallèle latin est d'autant plus net : une guerre contre des étrangers-ennemis (hostis), une dissension entre des amis et non-amis (inimicus). La réciprocité soulignée par Benveniste (hostire) prend alors tout son sens : on ne peut être ennemis (hostis) qu'entre étrangers (hostis), à quoi correspond la relation réciproque entre concitoyens, de civis à civis7. Impossible, donc, qu'un citoyen devienne ennemi, à moins qu'il soit étranger. Ajoutons que, selon cette logique, la guerre civile n'est pas une guerre de citoyens contre citoyens, mais de la cité contre elle-même, le rapport réfléchi se substituant au rapport réciproque dans les mots mêmes (Loraux, 1997, p.77).

Est-ce à dire que nous identifions là un invariant politique? Sans doute peut-on dire tout au plus que la politique de la cité a souvent rempli cette fonction d'entretenir la concorde, en refoulant jusque dans les mots la possibilité d'un conflit entre citoyens. Mais quelle place reste-t-il aux étrangers qui ne sont pas ennemis? Car, si l'ennemi est un étranger, rien n'indique que la réciproque soit systématique. Nous allons maintenant montrer comment la relation pratiquée avec cet étranger non-ennemi permet de mieux identifier les identités politiques grecque et romaine.

\section{Les pratiques (1) : la cité et les étrangers}

Dans le cas grec, la relation à l'étranger a plusieurs formes : la xenia puis la proxenia avec les étrangers extérieurs, la metoikia avec les métèques, les étrangers intérieurs. Nous l'avons dit, l'hospitalité grecque exclut la relation avec

7. Je ne peux donc être civis qu'à l'égard d'un autre civis, comme je suis nécessairement hostis à l'égard d'un hostis (Benveniste, 1970). 
un étranger véritablement étranger, car elle se constitue sur le modèle d'une relation réciproque, la xenia; celle-ci suppose la permutabilité des rôles (tantôt accueillant tantôt accueilli), donc une certaine équivalence des statuts ${ }^{8}$. L'étranger xénos est celui avec qui il est possible de contracter la xenia, soit l'hôte en puissance. D'où l'indistinction sémantique entre l'étranger et l'hôte, d'une part, et l'idée de proximité culturelle assurée par une langue commune, d'autre part : l'étranger est un hôte virtuel, l'hôte est un étranger actuel. La xenia est donc une forme d'alliance, une relation instituée impliquant droits et devoirs, familiale ou clanique, héréditaire et réciproque, appartenant au monde archaïque (précédant la création des poleis). Ce type d'hospitalité bien identifié par Moses Finley (1990, p.122-123) n'est ainsi ni strictement privé ni strictement public : il est précisément une relation intermédiaire, un contrat d'alliance qu'il ne faudrait pas simplement relayer à un moment pré-politique ou pré-juridique. À cette hospitalité archaïque de type «parenté artificielle » ou de «pseudo-parenté» (Herman, 1997) se juxtapose l'hospitalité de sens commun, privée, pratiquée individuellement et sans attente de réciprocité (inspirée par la pitié, elle se pratique généralement envers les plus pauvres).

L'évolution historique transforme la xenia en proxénia (autour du $6^{\mathrm{e}}$ siècle avant J.-C.), institution adaptée aux relations des citoyens des poleis; la proxénie substitue au lien familial d'alliance un lien impersonnel de nature politique. Le proxène, citoyen d'une cité choisie par une cité étrangère, a pour rôle de protéger, représenter et témoigner en faveur de ses citoyens. Il est la figure publique ou politique de l'hôte, un intermédiaire nécessaire pour suspendre la précarité juridique de l'étranger et le relier provisoirement à la cité (Vatin, 1984, p.15-21). Se démarque de ces pratiques une hospitalité religieuse (l'accueil des suppliants), suggérant déjà le double régime de l'hospitalité, entre justice et charité ; elle consiste à donner l'asile d'un sanctuaire à des individus, groupes réfugiés ou exilés politiques. La supplication est une demande rituelle de protection, c'est-à-dire une mise en relation immédiate avec la cité (par l'invocation de la protection divine), qui statuera sur la demande.

L'idée essentielle est qu'un étranger doit donc être préalablement reliéà un citoyen pour être reconnu, c'est-à-dire identifiable. C'est le rôle de témoin de l'hôte public, et la fonction plus générale des fameuses symbola, les archaïques tessères d'hospitalité devenues conventions judiciaires9. De la même façon, le métèque doit se choisir un patron (prostates) qui témoigne de cette qualité de

8. À défaut de pouvoir parler d'égalité stricte, voir Vatin, 1984, p. 144 et Boudou, 2012, p. 280-281.

9. Ces deux morceaux d'un même objet identifiant les partenaires d'une relation d'hospitalité sont connus notamment pour avoir servi de métaphores à Platon pour désigner les deux moitiés complémentaires du mythe de l'androgyne. Ces objets bien réels qu'on partage pour marquer et témoigner du contrat disparaissent en tant qu'objets, et sont remplacés par des contrats «papier» identifiés par un sceau (symbolon), puis plus généralement des conventions passées entre cités. Ils gardent le même nom en raison de l'imaginaire de complémentarité qu'ils suggéraient chez les Grecs. 
métèque devant les tribunaux. Il faut ainsi noter qu'avant d'être une catégorie - fiscale, juridique, (anti) civique -, la metoikia devrait se comprendre comme un type de relation. Car si le proxène a d'abord une fonction protectrice contre l'arbitraire du droit de saisie sur l'étranger, l'obligation du métèque de se relier à un prostates semble surtout impliquer la relation de dépendance, comme Aristote le suggère en comparant ces étrangers résidents à des «enfants » ou des «vieillards» (1993, III-1; Vatin, 1984, p. 184; Migeotte, 2004, p. 619).

Le métèque exprime le mieux l’ambiguïté politique de la cité grecque, à la fois définie par sa valeur et par un territoire. La polis est polysémique dès son invention, nommant tantôt la communauté de citoyens, tantôt la ville, le lieu d'habitation ou l'État (au sens d'une commune constitution) (Hansen, 2008). Mais l'élément crucial pour notre enquête est la séparation entre la frontière civique et les frontières territoriales, entre la concitoyenneté et la cohabitation. Aristote rappelle avec force que « la résidence ne fait pas la citoyenneté », tout comme l'accueil sur les terres ne présage en aucune manière de l'intégration dans le corps citoyen. "Oui à l'implantation, non à l'intégration », résume Henri Joly (1992, p.58), explicitant l'idée d'une citoyenneté antique exclusive et compréhensive fondée sur la liberté et l'autarcie (c'est-à-dire l'indépendance). Précisons néanmoins qu'il ne faudrait pas considérer la terre comme pure nécessité, simple actualisation de l'habitation indépendante de la communauté civique, comme le laisse entendre Aristote : l'exclusivité de la propriété foncière (réservée aux seuls citoyens) et l'idéologie de l'autochtonie irriguent largement la conception de la citoyenneté. À Athènes, l'ethnicisation de la citoyenneté, via la nécessité d'ascendance citoyenne affirmée par le décret de Périclès (451 avant J.-C.) puis, plus tard, l'interdiction des mariages mixtes (341 avant J.-C.) accompagnent l'idée que les Athéniens se caractérisent par leur enracinement. Contrairement aux « métèques », dont le premier sens, avant d'être le «co-habitant», est «celui qui transfère (meta) son oïkos d'une cité à une autre » (Gauthier, 1988, p. 27), les citoyens athéniens, «seul peuple à ne s’être pas déplacé », sont «nés de la terre» (Loraux, 1996; Détienne, 1990, 2003). Ce récit mythico-historique de l'enracinement raconte donc le fossé politique et axiologique entre le citoyen et le métèque, entre l'homme libre et autonome et le dépendant. Et si le citoyen est d'abord identifié par sa liberté, la communauté de citoyens est précisément celle qui affirme son autonomie par l'octroi souverain de la citoyenneté :

Il faut un décret de l'Assemblée qui représente la communauté tout entière. Seule la communauté des citoyens en exercice a le pouvoir d'agréger un nouveau membre [...]. Le nouveau citoyen, rangé dans une tribu, dans un dème, participe désormais de droit à une communauté politique indépendante, dont il recevra tout, charges et privilèges. (Gauthier, 1974, p. 209-210)

Le droit de cité est donc l'occasion d'affirmer et de pratiquer les valeurs 
fondamentales de la polis, il est l'expression de son identité politique, l'actualisation de ses principes - la liberté, l'autonomie, l'autarcie. Nous allons voir que ce sens de l'appartenance politique grecque est d'autant plus net par contraste avec les pratiques romaines.

\section{Les pratiques (2) : la citoyenneté extensive}

Le fameux édit de Caracalla (212 après J.-C.) est emblématique de la particularité romaine. Celui-ci distribue la citoyenneté romaine à tous les hommes libres de l'Empire, réalisant une conception extensive de la citoyenneté, marquée non pas par une sorte de générosité opposée à l'avarice grecque, mais par une identité politique expansionniste : «À Rome, on protège ses voisins, puis on les absorbe. L'étranger devient un ennemi, ou il devient Romain » (Gauthier, 1973, p. 21). Contrairement à une citoyenneté grecque accordée collectivement à des individus pour une intégration au sein d'un corps civique ${ }^{10}$, la citoyenneté romaine se diffuse par les cités conquises, selon la double appartenance impériale et locale, immortalisée par Cicéron (patrie de naissance et patrie de droit) (Thomas, 1995).

Il est inutile d'entrer dans les détails de l'octroi et des modalités de la citoyenneté romaine; cela est largement documenté et manquerait de pertinence directe pour notre enquête. Nous pouvons nous contenter de relever quatre éléments clivants, par rapport aux cas grecs : 1) tout d'abord, le fait qu'à partir des années 90 et 89 avant J.-C. (LexJulia et LexPlautia Papiria), la citoyenneté se distribue collectivement aux peuples libres italiens qui cesseraient de se révolter (Philippson, 1911, p. 255; Sherwin-White, 1939, p.132-133; Nicolet, 1976, p. 527) ; 2) bien souvent, des hommes sont faits citoyens romains sans avoir jamais vu Rome; on peut acquérir la citoyenneté via les petites cités et être ainsi romain partout, et inversement pérégrin toujours ${ }^{11}$;3) la citoyenneté romaine n'est pas seulement une affaire de droits, elle s'actualise par le statut social, lui-même indexé sur les relations; autrement dit, la citoyenneté est juridiquement individuelle et égalitaire mais politiquement discriminée, ou tout du moins graduée (Gauthier, 1974, p. 212-213) ; 4) le maître peut décider seul, en affranchissant son esclave, d'en faire un citoyen.

Pour synthétiser à gros traits, les Romains séparent donc plus nettement la citoyenneté de la cité, le droit de la politique (c'est-à-dire le statut de la

10. Nous mettons de côté les traités d'isopolitie, "par lesquels deux cités se donnaient réciproquement la citoyenneté potentielle, parfois en complément d'une alliance» (Migeotte, p. 626).

11. Aelius Aristide en fait ainsi l'éloge : «Vous ne divisez pas aujourd'hui les races en Grecs et barbares [...] vous avez remplacé [cette division] par la division en Romains et non-Romains. [...] Du fait de cette division, il y a dans chaque cité beaucoup d'hommes qui sont vos concitoyens tout autant que ceux de leurs compatriotes, bien que certains d'entre eux n'aient même pas encore vu Rome.» (Aristide, 1997, p. 91) 
participation), l'individu de la communauté. Cette évolution de la citoyenneté, de moins en moins politique, de plus en plus juridique, a bien sûr des causes multiples : les transformations morphologiques de la République à l'Empire appelèrent des modifications idéologiques et politiques considérables, notamment celle résumée par John Pocock (1995) par le passage du zoon politikon à l'homo legalis, ou plus généralement de la communauté «compréhensive » à la société de droit. Comme le dit Cicéron (1954, I-32), la civitas n'est pas plus qu'une societas. Mais c'est principalement la disparition relative des étrangers qui nous intéresse : les Romains divisent désormais le monde en Romains et non-Romains, division de droit plutôt que de culture, et la cité se fait de plus en plus «perméable» (Gauthier, 1974, p. 212). Plus besoin donc, d'une catégorie intermédiaire comme on la trouvait chez les Grecs, qui expliquait l'absence de tournant hostile du xénos.

Ce mouvement de lente cosmopolitisation du monde romain change la fonction de l'hospitalité. Elle s'institutionnalise, se juridicise, tout en héritant des modes d'alliance archaïques. La différence importante est qu'elle prendra un sens diplomatique et politique de grande ampleur, permettant à Rome de s'étendre en pactisant avec les cités voisines dans une relation mêlant l'hospitalité et le patronage (Nicols, 2011, p. 433; Étienne, Le Roux, Tranoy, 1987, p.333). L'hospitium privatum a pour forme une convention entre égaux, entre un citoyen romain et un étranger dont la cité est déjà partenaire de Rome, et pour fonction des services réciproques (protection, soutien en justice, etc.). Elle disparaît à la fin de la République, quand la multiplication des traités avec les cités étrangères assure la protection minimale des étrangers. C'est la relation client-patron qui lui succède (autour de la seconde moitié du $3^{\text {e }}$ siècle avant notre ère, il y a donc un chevauchement net), portant encore parfois le nom d'hospitalité. Elle opère entre citoyens romains de haut statut, servant d'intermédiaire (protection, justice) pour un peuple entier (Deniaux, 1987, p. 338). Enfin, c'est l'hospitium publicum, précédant l'institution du foedus ${ }^{12}$, qui sert à allier Rome avec une ville, un peuple ou des particuliers, faisant de l'hospitalité un outil diplomatique. Elle semble être tantôt un accord (d'amitié, de paix, réglant les relations privées des membres des deux cités entre eux) tantôt un signe d'honneur octroyé par Rome (Daremberg, Saglio, 1877-1919). Si cette tripartition n'a rien de systématique, notamment parce que l'hospitium se pratique sur environ 500 ans, on peut retenir la parenté avec les institutions grecques de ces «relations internationales», leur extension et leur politisation sans précédent.

12. Traité d'alliance entre les Romains et un autre peuple, notamment Barbare, ou entre peuples Barbares, incluant des considérations sur l'usage du territoire, les exemptions d'impôts, etc. Voir Sivan, 1987. 


\section{Critères pour l'étude des identités politiques}

Comprendre la place laissée à l'étranger permet ainsi de mieux identifier les identités politiques grecque et romaine, à savoir deux modèles ou matrices de l'appartenance, deux types de relation entre une communauté et les individus ou communautés extérieurs. L'intérêt de cette identification est qu'elle peut ne pas être simplement historique, à condition d'être bien conceptualisée. Encore une fois, le but n'est pas de découvrir des invariants; il s'agit plutôt de déterminer des idéaltypes politiques, heuristiques bien que façonnés historiquement. Si l'on suit le problème de la relation aux étrangers, et plus précisément des dispositifs d'exclusion et de définition relatifs aux unités d'action politique que sont Athènes (à partir du décret de Périclès de -451) et Rome (culminant avec l'édit de Caracalla de 212), nous pouvons identifier deux types d'appartenance, qui ne sont pas exclusives l'une de l'autre, mais hiérarchisées ou activées différemment. C'est ainsi que, bien qu'ayant une interprétation politique différente au problème général du mot hostis, nous poursuivons le travail d'Émile Benveniste.

En effet, dans une veine ouvertement structuraliste, le linguiste s'étonne de la symétrie inversée entre la polis grecque et la civitas romaine (Benveniste, 1970) : tandis que le mot politês (citoyen) est défini d'après le mot polis (cité), c'est à l'inverse le mot civitas (cité) qui est définie d'après le mot civis (citoyen). L'institution abstraite donne son nom au participant concret dans le premier cas, l'individu associé à d'autres détermine le nom du collectif dans le second. En somme, le tout détermine la partie chez les Grecs'13 (Veyne, 2005, p. 92), la partie détermine le tout chez les Romains. Le tout grec s'oppose à la collectivité romaine. Ces deux identités politiques distinctes dénotent deux types de relation aux étrangers, et, ce qui est mieux connu, deux formes de citoyenneté : la citoyenneté grecque est exclusive (parce qu'elle est conçue comme un privilège généralement inaccessible à quiconque n'est pas né citoyen), la citoyenneté romaine est inclusive. Autrement dit, si, comme nous l'avons dit, l'une et l'autre partagent la volonté de conjurer la guerre civile en concevant le conflit comme intrinsèquement (issu de l')étranger, elles ne distribuent pas les statuts des non-membres non-ennemis de la même façon, en raison d'une conception différente de la communauté.

Nous avons ainsi tenté de montrer comment Athènes s'identifie par un ensemble civique et une pluralité politique, Rome par une citoyenneté dynamique

13. Puisque la vie en cité est la fin de l'individu, elle en est sa nature. Selon l'argument téléologique d'Aristote, si la cité est bien un agrégat concentrique d'individus, de couples, de familles, de villages, elle leur est pourtant « antérieure » (Aristote, 1993, I-2). Il s'agit donc d'une antériorité (onto)logique, et non temporelle, la cité étant la raison d'être ordonnatrice de ces sousensembles, comme un corps l'est pour ses membres. 
et une unité juridique. Par « ensemble » il faut en entendre la connotation «finie» : une communauté forme un ensemble quand l'intégration d'éléments extérieurs est considérée comme une altération de l'identité de l'ensemble. Plus généralement, les traits que nous dégageons du modèle grec sont l'intensivité et l'exclusivité. La polis prend la forme d'une communauté définie par une ou des valeurs attribuée(s) de manière égale à chacun des membres, indépendamment de leur nombre et de leur situation géographique (critère d’intensivité). Ces valeurs forment une propriété finie qui exclut les éléments qui ne la partagent pas (critère d'exclusivité). Par contraste, on peut comprendre Rome comme une société dynamique : les éléments que nous dégageons du modèle romain sont l'extensivité et l'inclusivité. Rome prend la forme d'une collectivité extensive, c'est-à-dire d'un regroupement d'individus, de taille proportionnelle à ses éléments, supportant l'adjonction d'éléments étrangers sans craindre pour son unité (critère d'extensivité), se garantissant des droits réciproques sans limites a priori du cercle de réciprocité (critère d’inclusivité).

Ces concepts peuvent paraître abstraits. Cependant, ils permettent de poser les problématiques relatives aux identités politiques, quel que soit le contexte historique : considère-t-on que l'adjonction d'étrangers met en péril l'identité de la communauté? Y a-t-il des conditions culturelles qui doivent être remplies pour acquérir la citoyenneté ? Celle-ci est-elle ancrée territorialement? Finalement, au nom de quoi, de quelles valeurs politiques, justifie-ton les frontières?

\section{Références}

ARISTIDE Aelius, 1997, Éloges grecs de Rome, trad. L. Pernot, Paris, Les Belles Lettres.

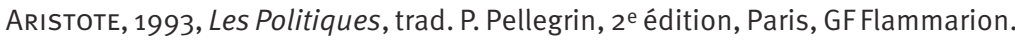

BenvenISTe Émile, 1969, Le vocabulaire des institutions indo-européennes, t. I : Économie, parenté, société, Paris, Minuit.

- 1970, "Deux modèles linguistiques de la cité », Échanges et communications. Mélanges offerts à Claude Lévi-Strauss, t. I, J. Pouillon et P. Maranda éd., Paris La Haye, Mouton, p. 589-596.

Boudou Benjamin, 2012, "Éléments pour une anthropologie politique de l'hospitalité », Revue du Mauss, nº 40, p. 267-284

CICÉRON, 1954, De la République, trad. C. Apuhn, Paris, Garnier.

- 1974, Les devoirs, trad. M. Testard, Paris, Les Belles Lettres.

Daremberg Charles-Victor, SAglio Edmond, 1877-1919, Dictionnaire des Antiquités grecques et romaines, Paris, Hachette.

Denıaux Élisabeth, 1987, "Les hôtes des Romains en Sicile», Sociabilité, pouvoirs et société, F. Thelamon éd., Rouen, Publications de l'Université de Rouen.

DERRIDA Jacques, 1996, Le monolinguisme de l'autre, Paris, Galilée.

- 1997, Adieu à Emmanuel Lévinas, Paris, Galilée. 
Derrida Jacques, Dufourmontelle, Anne, 1997, De l'hospitalité, Paris, Calmann Lévy. DÉtIEn ne Marcel, 1990, «Qu'est-ce qu’un site?», Tracés de fondation, M. Détienne éd., Louvain-Paris, Peeters.

- 2003, Comment être autochtone. Du purAthénien au Français raciné, Paris, Le Seuil. DumÉZIL Georges, 1977, Les dieux souverains des Indo-Européens, Paris, Gallimard.

ÉTIEN ne Robert, LE Roux Patrick, TRAnOY Alain, 1987, «La Tessera Hospitalis, instrument de sociabilité et de romanisation dans la péninsule ibérique », Sociabilité, pouvoirs et société, F. Thelamon éd., Rouen, Publications de l'Université de Rouen.

Finley Moses, 1990, Le monde d'Ulysse, trad. C. Vernant-Blanc et M. Alexandre, Paris, La Découverte.

GALLEGo Julie, 2010, «L'hôte et l'ennemi sont-ils des étrangers comme les autres?», Figures de l'étranger autour de la Méditerranée antique. À la rencontre de l'Autre, M.-F. Marein, P. Voisin éd., Paris, L'Harmattan.

GAUthIER Philippe, 1972, Symbola. Les étrangers et la justice dans les cités grecques, Nancy, Annales de l'Est.

- 1973, « Notes sur l'étranger et l'hospitalité en Grèce et à Rome », Ancient Society, vol. IV.

- 1974, "Générosité" romaine et "avarice" grecque. Sur l’octroi du droit de cité», Mélanges d'histoire ancienne offerts à William Seston, W. Seston éd., Paris, E. de Boccard.

- 1988, "Métèques, périèques et paroikoi. Bilan et points d'interrogation », L'étranger dans le monde grec, R. Lonis éd., Nancy, Presses universitaires de Nancy.

Grangé Ninon, 2003, "Cicéron contre Antoine. La désignation de l'ennemi dans la guerre civile», Mots. Les langages du politique, nº 73 .

- 2009, De la guerre civile, Paris, Armand Colin.

HANSEN Mogens, 2008, Polis. Une introduction à la cité grecque, trad. F. Regnot, Paris, Les Belles Lettres.

HÉnAFF Marcel, 2002, Le prix de la vérité, Paris, Le Seuil.

Herman Gabriel, 1987, Ritualised Friendship and the Greek City, Cambridge, Cambridge University Press.

- 1997, "Le parrainage, l'“hospitalité” et l'expansion du christianisme», Annales, vol. LII, n6, p. 1305-1338.

Joly Henri, 1992, Études platoniciennes. La question des étrangers, Paris, Vrin.

KoSELLECK Reinhart, 1990, Le futur passé. Contribution à la sémantique des temps historiques, trad. J. et M.-C. Hook, Paris, EHESS.

Loraux Nicole, 1996, Né de la Terre. Mythe et politique à Athènes, Paris, Le Seuil.

- 1997, La cité divisée. L'oubli dans la mémoire d'Athènes, Paris, Payot.

MAIER Christian, 1984, Introduction à l'anthropologie politique de l'Antiquité classique, trad. P. Blanchaud, Paris, PUF.

MigeOTTE Léopold, 2004, "La mobilité des étrangers en temps de paix en Grèce ancienne", La mobilité des personnes en Méditerranée de l'Antiquité à l'époque moderne. Procédures de contrôle et documents d'identification, C. Moatti éd., Rome, École française de Rome.

Nicols John, 2011, «Hospitality among the Romans», The Oxford Handbook of Social 
Relations in the Roman World, M. Peachin éd., New York, Oxford University Press. NiCOLET Claude, 1976, Le métier de citoyen dans la Rome républicaine, Paris, Gallimard. Philippson Coleman, 1911, The International Law and Custom of Ancient Greece and Rome, Londres, Macmillan.

Platon, 2008, République, Éuvres complètes, L. Brisson éd., Paris, Flammarion.

Pocock John, 1995, "The ideal of citizenship since classical times», Theorizing Citizenship, R. Beiner éd., Albany, State University of New York Press.

Schmitt Carl, 1992, La notion de politique suivi de Théorie du partisan, trad. M.-L. Steinhauser, Paris, Flammarion.

SherWIn-White Adrian, 1939, The Roman Citizenship, Oxford, Oxford University Press.

SIVAN Hagith, 1987, "On Foederati, Hospitalitas, and the settlement of the Goths in A.D. 418 ", The American Journal of Philology, vol. CVIII, nº 4, p. 759-772.

THomas Yan, 1995, "Le droit d'origine à Rome. Contribution à l'étude de la citoyenneté », Revue critique de droit international privé, vol. LXXXIV, nº 2, p. 253-288.

VATIN Claude, 1984, Citoyens et non-citoyens dans le monde grec, Paris, CDU et SEDES.

VeYNe Paul, 2005, L'empire gréco-romain, Paris, Le Seuil. 